\title{
BOCHALEMA - SISTEMA DE ALERTA TEMPRANA
}

\author{
Laverde P Luz Amanda., Rivera María Esther
}

\begin{abstract}
Facultad de Ingenierías y Arquitectura. Programa Ingeniería Ambiental. Grupo de Investigaciones Ambientales Agua, Aire y Suelo (GIAAS). Universidad de Pamplona. Pamplona. Colombia Ingeniero Ambiental. Grupo de Investigaciones Ambientales Agua, Aire y Suelo (GIAAS). Universidad de Pamplona. Pamplona. Colombia.
\end{abstract}

\section{Resumen}

Es importante destacar que el municipio de Bochalema no cuenta con sistemas de alertas tempranas, además no cuentan con acciones en prevención y mitigación del riesgo, siendo factor preponderante en la presencia de escenarios del riesgo y población afectada por fenómenos naturales. Por lo tanto, el objetivo del proyecto es diseñar el Sistemas de Alerta Temprana (SAT) para Bochalema - proyecto piloto, específicamente en las quebradas Aguablanca y La Chiracoca, convirtiéndose en herramienta base valiosa que obtenga documentación para el municipio sobre los eventos que se puedan presentar. Con la creación del Sistema Nacional de Gestión del Riesgo de Desastre, se garantiza la aplicación de la ley 1523 del 2012 donde los municipios están obligados a crear los consejos municipales para la gestión del riesgo, planteando las estrategias y medidas a realizar en caso de emergencias o cualquier tipo de fenómeno amenazante. Para ello, se realizó un análisis técnico, ambiental y social, levantamiento de información hidrometeorológica, y comunitaria de la zona de estudio, se estimó el comportamiento de las precipitaciones máximas (curvas IDF) y caudales máximos aplicando análisis estadístico, mediante el software Hec-Ras se determinaron las planicies de inundación, por último se realizó el montaje institucional y operativo de SAT en el municipio, obteniendo la zona apta para estación. Se concluye que los fenómenos naturales de riesgo con una mayor incidencia en la zona de estudio son los de origen hidrometeorológico seguido por los de origen geológico, así como también se presentan amenazas por fenómenos antropogénicos como la minería.

Palabras clave: Bochalema, Curva idf, Planicies de inundación, Sistema de Alertas Tempranas, Hec - Ras

\section{BOCHALEMA - EARLY WARNING SYSTEM}

\section{Abstract}

Importantly Bochalema municipality has no Early Warning Systems, also have not actions in prevention and mitigation of the risk, being important factor in the presence of scenarios risk and population affected by natural phenomena. Therefore, the objective of the project is to design the Early Warning System 
(SAT) to Bochalema - pilot project, specifically in the catchment Aguablanca and La Chiracoca, becoming specially tool for basis documentation for the town about events that may present. With the creation of the National System for Disaster Risk Management, the application of the law 1523 of 2012 the municipalities need to create the councils municipal for risk management, raising the strategies and measurements to realize in case of emergencies or any type of phenomenon. For this, a community technical, environmental and social analysis, raising of hydrometeorological information, and the study's area, the behavior of the maximum rainfall (IDF curves) and peak flows are estimated using statistical analysis software by HEC Ras floodplains were determined finally the institutional and SAT institutional and operative SAT assembly in the municipality, obtaining the suitable area for station. Concluded that natural phenomena risk with a higher incidence in the study's area are followed by hydro-meteorological disasters of geological origin and threats are also presented by anthropogenic phenomena such as mining.

Keywords: Bochalema, idf Curv, Flooding, Early Warning Systems, Hec-Ra

Para citar este artículo: Laverde P Luz Amanda., Rivera María Esther.Bochalema-sistema de alerta temprana.Revista Bistua.2015.13(2):91-111

+ Autor para el envió de correspondencia y la solicitud de las separatas:Maria Ester Rivera.Universidad de Pamplona.Facultad de Ingenierias y Arquitectura.Programa de Ingenieria Ambiental. Grupo de Investigaciones Ambientales Agua, Aire y Suelo (GIAAS).email: maes@unipamplona.edu.co 


\section{Introducción}

El área de estudio se encuentra situada en el municipio de Bochalema en la Sub-región SurOriental del Departamento Norte de Santander ${ }^{1}$. Su cabecera municipal está localizada a los 70 $37^{\prime}$ de latitud norte y $72^{\circ} 39^{\prime}$ de longitud oeste del meridiano de Greenwich. El territorio municipal limita por el norte con San Cayetano y Cúcuta, por el oriente con Los Patios y Chinácota, por el sur con Pamplonita, por el suroccidente con Cucutilla y por el occidente con Arboledas y Durania, (Ver imagen 1)

\section{Imagen 1. Ubicación del Municipio} de Bochalema

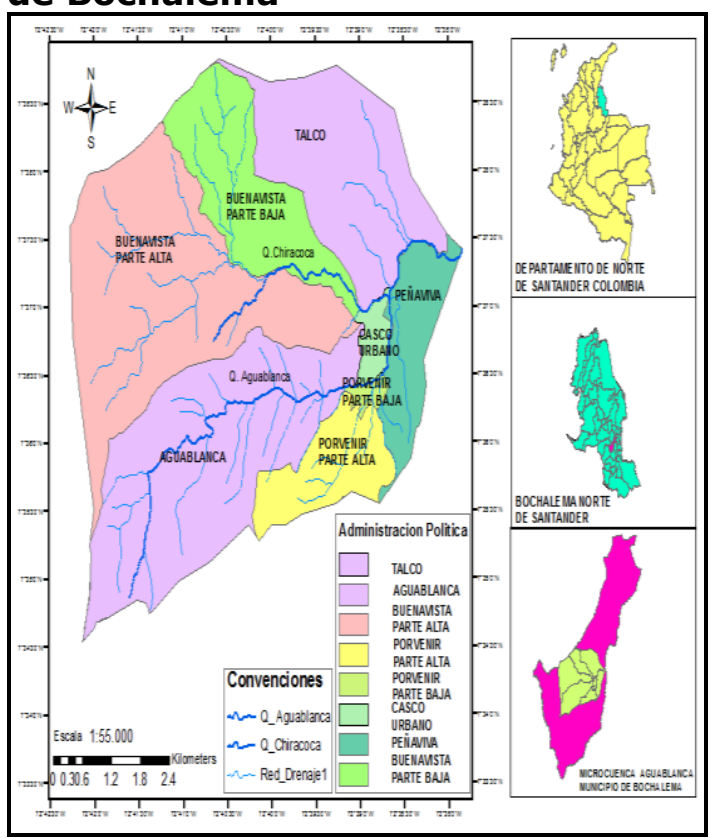

Fuente: Autores

Los Sistemas de Alerta Temprana conocidos como SAT, son un conjunto de procedimientos e instrumentos, a través de los cuales se monitorea una amenaza - evento adverso de carácter previsible, se recolectan y procesan datos e información, 93 ofreciendo pronósticos o predicciones temporales sobre su acción y posibles efectos. Millones de personas en todo el mundo salvan sus vidas y sus medios de subsistencia gracias a la implementación de estos sistemas y su objetivo principal es dar el poder a personas y comunidades amenazadas de actuar a tiempo y de manera apropiada para reducir la posibilidad de heridas, pérdida de vida, daño a la propiedad y al ambiente y pérdida del sustento ${ }^{2}$. Cada una de las agencias gubernamentales como los servicios meteorológicos nacionales, agencias nacionales y locales de gestión de desastres y las organizaciones no gubernamentales, empresas, instituciones académicas, socios internacionales y comunidades locales juegan un papel esencial en el diseño y la implementación exitosa de la mayoría de los sistemas de alerta temprana de amenazas naturales.

En el Manual para el diseño e implementación de un sistema de alerta temprana de inundaciones en cuencas menores se propone la creación de un método sistemático que reúna los aspectos clásicos de los programas de preparativos y respuesta a emergencias con propuestas para reducir la vulnerabilidad y el registro de los regímenes hídricos para alertar a la comunidad de las posibilidades de inundaciones. De igual manera, la capacitación y apoyo de grupos de ciudadanos y profesionales de organizaciones públicas y privadas, y organizaciones no gubernamentales en comunidades 
locales en el análisis de vulnerabilidad a inundaciones $y$ desarrollo de medidas de mitigación, monitoreo de eventos hídricos y meteorológicos, desarrollo sistemas de alerta local, y desarrollo de programas de preparativos y respuesta a emergencias de inundaciones ${ }^{3}$. Mientras que UNESCO en su documento Conceptos y Herramientas Sobre sistemas de Alerta Temprana y Gestión del Riesgo para la Comunidad Educativa contiene una serie de herramientas, conceptos y recomendaciones para fortalecer la participación de la comunidad educativa en la gestión de riesgos y los sistemas de alera temprana de las comunidades amenazadas, permitiendo la identificación y descripción de los diferentes SAT que tenemos en el país, sugiriendo mecanismos de participación, aportes y compromisos del centro educativo.

En la Guía de referencia para sistemas de alerta temprana de crecidas repentinas del Departamento administrativo oceánico de los Estados Unidos ${ }^{4}$ proporciona una descripción de los requisitos operativos y organizacionales para un sistema de pronóstico de crecidas repentinas dentro del marco de un sistema de alerta temprana multiamenaza. Además de servir de orientación para el desarrollo de un concepto de operaciones para el sistema de alerta temprana de crecidas repentinas.

En Colombia existen zonas vulnerables y susceptibles a eventos extremos ocasionados por las lluvias y el Valle de Aburrá no es ajeno a esta situación, además por sus complejas condiciones físicas en el territorio, sumadas a los altos índices de vulnerabilidad ante la ocurrencia de fenómenos de origen natural tales como movimientos en masa, los cuales representan el $35 \%$ de los eventos que ocurren en el valle y el $77 \%$ de las víctimas mortales. Debido a esta grave problemática, desde el año 2008 se ha venido utilizando un sistema de alerta temprana por movimientos en masa inducidos por lluvia soportando umbrales de lluvia empíricos y proporcionando un rápido medio para monitorear y comunicar información sobre amenazas a una comunidad vulnerable, brindado tiempo para tomar acciones que pueden reducir las condiciones de riesgo ${ }^{5}$.

El diseño del Sistema de Alerta Temprana (SAT) debe estar centrado en la población, para dar procesos de participación comunitaria orientados a fortalecer la educación de las personas involucradas en la gestión local del riesgo, aportando finalmente la reducción de la vulnerabilidad de las comunidades. Atendiendo a fenómenos de deslizamientos e inundaciones, este SAT meditó que los fenómenos de deslizamientos no se consideran sujetos a predicciones, mecanismos o procedimientos que permitan conocer los sectores afectados por estos fenómenos con anticipación, debido a las limitaciones logísticas y tecnológicas del Departamento de Risaralda para la 
implementación de modelos de predicción de deslizamientos ${ }^{6}$

De igual manera, en la Guajira se llevó a cabo el proyecto Diseño de un sistema de alerta temprana por inundación y deslizamiento en el flanco nororiental de la Sierra Nevada de Santa Marta, planteando la instalación de una red de estaciones automáticas hidrometeorológicas para conocer el comportamiento de la precipitación y niveles de la superficie del agua en los drenajes en tiempo real, y así prevenir o disminuir mediante la detección y emisión de alertas tempranas la pérdida de vidas humanas $\mathrm{y} / \mathrm{o}$ bienes materiales, como también las actividades económicas (agricultura), anticipándose a la ocurrencia de inundaciones y fenómenos de remoción en masa. Este SAT se propone formar parte las medidas de prevención o mitigación de problemas, generados por fenómenos de inundación y deslizamientos de tierra, detonados principalmente por intensas lluvias y/o actividades antrópicas, por lo tanto el SAT no representa una solución definitiva a los problemas del sector pero si una primera aproximación, que una vez calibrada permitirá la toma de decisiones oportunas en criterios mejor sustentados ${ }^{7}$.

Para el diseño e implementación de un Sistema de Alerta Temprana, se debe considerar una serie de elementos y de estructuras multisectoriales y multi-institucionales, además otros componentes que determinan su aplicación y éxito. Algunas organizaciones internacionales identifican 5 elementos fundamentales que deben ser tomados en cuenta para la creación de un SAT:

1. Organización Comunitaria: Formación de un comité organizador y reunión inicial con miembros de la comunidad para formar equipos de trabajo y explicar conceptos generales para el desarrollo del Programa. El comité organizador debe estar compuesto por líderes de la comunidad: Organizaciones no gubernamentales (ONG), organizaciones voluntarias, clubes o asociaciones de la comunidad, El sector público: la municipalidad, oficinas locales del gobierno, empresas del Estado (electricidad, agua, etc.), el sector privado: industrias, empresas, agricultores, negocios en general.

2.-Reconocimiento de la Cuenca (Existencia y conocimiento del riesgo): Elaboración del mapa de la cuenca, visualización del concepto de cuenca, elaboración del mapa de la comunidad, análisis del problema, se debe identificar las amenazas y tener conocimiento de los riesgos, o eventos potencialmente peligrosos que puedan afectar a las poblaciones, infraestructuras y recursos expuestos al impacto de dichos fenómenos, ubicándolo en un Mapa de Riesgo, debido a que conociendo las amenazas, vulnerabilidades y los elementos expuestos a dichos fenómenos, se puede estimar la potencialidad del peligro y los daños que se puedan generar, para tomar medidas de Gestión de Riesgo como los Sistemas de Alerta Temprana. 
3. Medición de Lluvia y Nivel de Agua de los Ríos: La medición de la lluvia y del nivel de agua de los ríos y quebradas tiene por objetivo hacer un seguimiento a las condiciones hidrológicas que pueden producir una inundación.

Según la Organización
Meteorológica Mundial (OMM) al momento de instalar una estación debe considerar: - Evitar la cercanía a grandes fuentes de calor industrial, techos, cuestas empinadas, vegetación alta, pantanos, lugares bajos sujetos a encharcamiento después de eventos de lluvia, y en la vecindad de escapes de aire. - Para mediciones de vientos se recomienda para agricultura y otras aplicaciones a $2 \mathrm{~m}$ sobre el suelo, aunque para fines sinópticos se realiza a $10 \mathrm{~m}$. Se recomienda cobertura del suelo de pasturas bajas, no arvenses. - Altura de sensores de radiación, brillo solar y precipitación no tienen normas específicas porque no responden a un gradiente vertical. - Revisar con frecuencia el pluviómetro para ver que no tenga agujeros en ninguna de sus partes, esté libre de tierra, insectos o basuras y que esté firme y bien nivelado. - La temperatura del aire ambiental y la humedad relativa se mide generalmente entre 1,5 y $2 \mathrm{~m}$ sobre el suelo. $(1.75-1.8 \mathrm{~m})$. - Los sensores de temperatura y humedad se sitúan de tal manera que el obstáculo u obstrucción más cercana esté a una distancia superior a cuatro veces su altura, y al menos a $30 \mathrm{~m}$ de grandes áreas, o carreteras.

4. El funcionamiento del sistema de alerta propiamente dicho consiste en las siguientes actividades ${ }^{8}$ :

a. Lectura y Registro: Cuando el fenómeno monitoreado produce alguna alteración, activación o manifestación de peligro, es registrado por los instrumentos, y se procede a tomar las lecturas correspondientes, manteniendo una vigilancia pormenorizada, continua y permanente para conocer sus cambios y evolución.

b. Transmisión de Datos: Luego registrar las lecturas, son transmitidas inmediatamente, para que los encargados o especialistas efectúen los cálculos necesarios y se realicen pronósticos respectivos sobre la posible ocurrencia o no de un evento adverso o destructivo.

c. Procesamiento y Análisis de Datos: Los datos llegan a manos de expertos 0 encargados de procesarlos, quienes realizan sus cálculos para la búsqueda de información hidrológica, análisis de la información histórica de precipitación y establecer si estos indican la posibilidad 0 no de manifestarse un evento adverso o destructivo.

d. Evaluación de la Situación y Definición de la Alerta: LaS instituciones encargadas 0 los miembros de los comités de Emergencias de las comunidades, evalúan la información 0 el resultado del análisis de los datos procesados y lo contrastan con un Mapa de Riesgo, determinando así el daño potencial, nivel y tipo de alerta que se debe declarar y emitir.

e. Difusión de la Alerta: Al contar con la alerta oficial debidamente 
definida, emitida y comprobada, se procede a notificarla a la población. La alerta se podrá difundir utilizando radios de comunicación, radio emisoras, teléfonos, radio parlantes, bocinas, sirenas, banderas, sonando pailas, campanas y cualquier otro instrumento que tenga el alcance, que permita informar rápidamente a la comunidad.

f. Activando el Plan de Emergencias o Respuesta: Sin este paso la alerta, no tendría sentido o ningún resultado, por lo tanto es imprescindible que todos los centros educativos y las comunidades cuenten con planes o actividades de preparación para respuesta.

5. La Evaluación de la Situación, Difusión de la Alerta y Plan de Emergencia: Aviso de alerta a toda la comunidad cuando hay peligro de inundación. Cualquier comunidad que es vulnerable a inundaciones debe siempre contar con un plan de emergencia para así saber responder ante un posible peligro de inundación. Este plan consiste en haber pensado de antemano en una serie de medidas que tienen por finalidad brindar seguridad a la población.

En el Análisis Estadístico aplicada a la información hidrológica es necesario considerar que es la variable aleatoria " $X$ " es una variable descripta por una "distribución de probabilidad" y ésta determina la posibilidad de que una observación " $x$ " de la variable " $X$ ", caiga en un rango preestablecido de " $X$ " 9 . Por ejemplo, si "X" es la "precipitación anual" en un lugar determinado, entonces la "distribución de probabilidad de $X^{\prime \prime}$ determina la posibilidad de que la precipitación anual observada en una año dado, "xi", caiga en un rango definido, tal como "menos de $200 \mathrm{~mm}$ ", o "entre 200 y $250 \mathrm{~mm}^{\prime \prime}$ y sucesivamente.

En los países tropicales las lluvias son irregulares, con gran variabilidad espacio-temporal, pero tienen la propiedad de persistencia a largo plazo, de manera que episodios de precipitaciones intensas están seguidos a su vez de otros similares, existiendo una correlación positiva entre ellos ${ }^{10}$. Por esta razón, las precipitaciones presentes y pasadas pueden utilizarse para predecir precipitaciones en el futuro. Las medidas obtenidas en las estaciones determinan el estado del sistema o comportamiento del cambio climático; por lo tanto, la serie reconstruida puede servir como referencia para pronosticar un estado futuro del sistema en una estación local, regional o nacional.

Adicionalmente al problema del análisis de series de precipitaciones en un entorno complejo, se tiene el problema de los datos faltantes y la calidad de la base de datos ${ }^{11}$. Es común encontrar una gran cantidad de registros con poca duración, numerosos errores y con ausencia de datos. La calidad en la base de datos es una información clave para detectar y monitorear la variabilidad en el clima. Para ello, se utilizan diferentes métodos como: Proporciones normales con 
estaciones vecinas o con la misma estación, Group means, Hot, Deck, Predicted mean imputation, Predictive model based, Propensity score method, Predictive mean matching method, Mahalanobis distance $y$ Propensity score/predictive mean/Mahalanobis distance combination method. En esta oportunidad se aplicara el método de proporciones normales.

Método de proporciones normales es uno de los más utilizados en aquellos casos en que no existen datos de comparación y por tanto, la serie tiene que servir de referencia para el relleno de datos faltantes de sí misma. Cuando se desconoce la lluvia caída de un mes cualquiera, se establece una razón de proporcionalidad entre la lluvia mensual y anual como se muestra en la Ecuación 1.

\section{$\frac{X}{x}=\frac{P f}{P a-X} \quad$ Ecuacion 1}

Dónde, $\quad X$ - Lluvia del mes faltante, $\quad x$ - Lluvia promedio del mes faltante, Pf - Total anual (del mes faltante), $\quad P a$ - Total anual promedio

Las Pruebas de bondad de ajuste consisten en comprobar, gráfica y estadísticamente, si la frecuencia empírica de la serie analizada se ajusta a una determinada función de probabilidades teórica seleccionada a priori, con los parámetros estimados con base en los valores muéstrales. Las pruebas de bondad de ajuste más utilizadas son: a. Ajuste gráfico y $b$. Ajuste estadístico: Chi - cuadrado $\left(X^{2}\right)$ y Kolmogorov - Smirnov (K-S)
La prueba Chi - cuadrado se basa en el cálculo de frecuencias tanto de valores observados, como valores esperados, para un número determinado de intervalos. Esta prueba es comúnmente usada para verificar la bondad de ajuste de la distribución empírica a una distribución teórica conocida. Fue propuesta por Karl Pearson en $1900^{12}$. La expresión general de la prueba Chi - cuadrado está dada por la Ecuación 2.

$X_{C}^{2}=\sum_{i=1}^{K} \frac{\left(\theta_{i}-e_{i}\right)^{2}}{e_{i}}$

Ecuacion 2

Dónde,

$\sum_{i=1}^{K} \theta_{i}=\sum_{i=1}^{K} e_{i}=N$

$X_{C}^{2}$-Valor cuadrado de Chi cuadrado a partir de los datos, $\theta_{i}$ - Número de valores observados en el intervalo de clase i, $e_{i}$ Número de valores esperados en el intervalo de clase i, $\quad K$ - Número de intervalos de clase.

Asignando igual probabilidad de ocurrencia a cada intervalo de clase, se obtiene la ecuación 3 :

$\boldsymbol{X}_{C}^{2}=\sum_{i=1}^{K} \frac{\left(\boldsymbol{N}_{i}-N P_{i}\right)^{2}}{N P_{i}}$

Ecuacion 3

Dónde $\mathrm{N}_{i}$ - Número de observaciones que caen dentro de los límites de clases ajustados del intervalo i, $N$ - Tamaño muestral, $P_{\bar{i}}$ - probabilidad igual para todos los intervalos de clase y $P_{i}=$ $1 / \mathrm{K} \circ \mathrm{e}_{\mathrm{i}}=\mathrm{P}_{\mathrm{i}} \mathrm{N} \quad$ Simplificando la Ecuación 3 se obtiene la fórmula computacional desarrollada por Markovic (1965) está dada por la Ecuación 4. 
$X_{C}^{2}=\frac{K}{N} \sum_{i=1}^{K} N_{i}^{2}-N$

Ecuacion 4

El valor de $X_{C}^{2}$ obtenido de la Ecuación 4 se compara con el $\mathrm{X}_{\mathrm{t}}^{2}$ cuyo valor se determina con: Nivel de significación: $\alpha=0,05$ ó $\alpha=0,01$ y Grados de liberad: $\mathrm{g} . \mathrm{l}=\mathrm{k}-1-\mathrm{h} \quad$ donde $\mathrm{h}=$ Número de parámetros a estimar. El Criterio de Decisión, Si $X_{C}^{2} \leq \mathrm{x}_{\mathrm{t}}^{2}$ entonces se acepta la hipótesis de que el ajuste es

Bueno al nivel de significación 0,05 .

\section{La Prueba de Smirnov - Kolmogorov compara las} diferencias existentes entre la probabilidad empírica de los datos de la muestra y la probabilidad teórica, tomando el valor máximo del valor absoluto de la diferencia entre el valor observado y el valor de la recta teórica (ver Ecuación 5) del modelo ${ }^{12}$.

\section{$\Delta=$ máx $|F(x)-P(x)| \quad$ Ecuacion 5.}

Dónde $\Delta-$ Estadístico de Kolmogorov - Smirnov, cuyo valor es igual a la diferencia

Máxima existente entre la probabilidad ajustada y la probabilidad empírica, $\mathrm{F}(\mathrm{x})$ probabilidad de la distribución teórica, $P(x)$ - Probabilidad experimental o empírica de los datos (frecuencia acumulada).

En el Criterio de decisión se comparan los valores estadísticos, con el valor crítico, con los siguientes criterios de decisión: $\Delta<\Delta \mathrm{o} \rightarrow \mathrm{EL}$ ajuste es bueno, al nivel de significación seleccionado.

El análisis de frecuencia ${ }^{12}$ es una herramienta utilizada para predecir el comportamiento futuro de los caudales en un sitio de interés, a partir de la información histórica de caudales. Su confiabilidad depende de la longitud y calidad de la serie histórica, además de la incertidumbre propia de la distribución de probabilidades seleccionada. Cuando se pretende realizar extrapolaciones, período de retorno mayor que la longitud de la serie disponible, el error relativo asociado a la distribución de probabilidades utilizada es más importante, mientras que en interpolaciones la incertidumbre está asociada principalmente a la calidad de los datos a modelar; en ambos casos la incertidumbre es alta dependiendo de la cantidad de datos disponibles.

Periodo de retorno (Tr) - Intervalo promedio de tiempo en años, dentro del cual un evento de magnitud $x$ puede ser igualado 0 excedido, por lo menos una vez en promedio. Así, si un evento igual o mayor a $x$, ocurre una vez en $T$ años, su probabilidad de ocurrencia $P$, es igual 1 en $T$ casos, como se expresa en la Ecuación 6.

$T=\frac{1}{P\left(x \geq x_{T}\right)}$

Ecuacion 6

Dónde $P\left(x \geq x_{T}\right)$ - Probabilidad de ocurrencia de un evento $\geq x, T$ Período de retorno.

La función de distribución de probabilidad con la cual los 
caudales máximos se ven mayormente reflejados, es la función de Gumbel, considerando su uso como altamente confiable, entregando un coeficiente de determinación promedio para todas las estaciones del $96,4 \%$ y una aprobación altamente significativa de la prueba de bondad de ajuste Kolmogorov - Smirnov.

Una vez seleccionada la fórmula empírica a utilizar, se procede a ordenar los datos de la muestra de menor a mayor, después se les asigna la probabilidad empírica, que es la probabilidad de no excedencia.

En la tabla 1 se aprecias las fórmulas empíricas propuestas por diferentes autores para calcular dicha probabilidad.

Tabla 1. Fórmulas de
probabilidades empíricas

\begin{tabular}{|l|l|}
\hline Formula Empírica & \multicolumn{1}{|c|}{ Probabilidad } \\
\hline HAZEN (1930) & $\begin{array}{l}\boldsymbol{P}(\boldsymbol{x})=\mathbf{( m - 0 . 5 )} \\
\mathbf{n}\end{array}$ \\
\hline $\begin{array}{l}\text { CHEGODAYEV } \\
(1955)\end{array}$ & $\begin{array}{l}\boldsymbol{P}(\boldsymbol{x})=(\mathbf{m}-\mathbf{0 . 3}) \quad / \\
(\mathbf{n}-\mathbf{0 . 4})\end{array}$ \\
\hline WEIBULL (1939) & $\boldsymbol{P}(\boldsymbol{x})=\mathbf{m} / \mathbf{( n + 1 )}$ \\
\hline $\begin{array}{l}\text { CALIFORNIA } \\
\text { (1923) }\end{array}$ & $\boldsymbol{P}(\boldsymbol{x})=(\mathbf{m}) / \mathbf{n}$ \\
\hline $\begin{array}{l}\text { Dónde } m \text { - Número de orden o posición } \\
\text { y } n \text { - Número total de datos }\end{array}$ \\
\hline
\end{tabular}

Fuente: Villón, M, 2006

La distribución Gumbel es una de las distribuciones de valor extremo, llamada también Valor Extremo Tipo I, Fisher-Tippett tipo I o distribución doble exponencial ${ }^{13}$.

La función de distribución acumulada de la distribución Gumbel, tiene la forma de la Ecuación 7.
$F(x)=\operatorname{EXP}\left(-\operatorname{EXP}\left(-\frac{x-\mu}{\alpha}\right)\right) \quad$ Ecuacion 7

Dónde $F(x)$ - Función de densidad de Gumbel de la variable $x, \alpha$ Parámetro de escala, también desviación estándar, $x$ - Variable independiente, $\mu$ - Parámetro de posición, también llamado moda. Los parámetros a y $\mu$ se estiman, tal como se muestra en las ecuaciones 8 y 9.

$\alpha=\frac{\sqrt{6}}{\pi} * S \quad$ Ecuacion 8

$\mu=\bar{x}_{\imath}-0,5772 * \alpha$

Ecuacion 9

Dónde $S$ - Desviación estándar, $\overline{x_{2}}$ - Parámetros de la media Con el análisis probabilístico llevado a cabo con lluvias máximas diarias anuales cuyos registros se toman a un intervalo fijo y único de observación, que se relacionan mediante un coeficiente prácticamente independiente del lugar y del periodo de retorno con las lluvias de duración de 24 horas, el coeficiente de corrección por intervalo fijo de observación toma un valor aproximado a 1.13, es decir, que los registros realizados a intervalos fijos subestiman la precipitación real considerando su misma duración. Para convertirlos a registros de duración de 24 horas se les debe afectar por el valor del coeficiente indicado. El índice de corrección por intervalo fijo de observación indicado llega a ser un primer coeficiente de desagregación que transforma lluvias de duración diaria a lluvias de duración de 24 horas $^{14}$.

Las curvas intensidad-duraciónfrecuencia (IDF) como relación que 
existe entre la intensidad de precipitación media y la frecuencia de ocurrencia; estas curvas son herramientas ampliamente utilizadas en ingeniería para fines de planeación, diseño y operación de los proyectos hidráulicos, así como para la protección de obras de ingeniería contra avenidas máximas $^{15}$. De acuerdo a ${ }^{16}$ el análisis de frecuencia de la precipitación es usado ampliamente para diseño de obras de ingeniería que controlan los escurrimientos generados por las tormentas. Esto incluye sistemas de alcantarillado municipales, vertedores, puentes y sistemas de drenaje agrícola. Mientras 17 menciona la relación intensidadduración-frecuencia de precipitaciones extremas es ampliamente usada para estimar las avenidas de diseño en los sitios donde se construirán las obras hidráulicas.

Intensidad (I) se define como el volumen de precipitación o altura equivalente de precipitación por unidad de tiempo $(\mathrm{mm} / \mathrm{h})$ como se expresa en la Ecuación $10^{18}$.

$\boldsymbol{I}=\frac{\boldsymbol{P}}{\boldsymbol{T d}}$

Ecuacion 10

Donde $\quad I$ - Intensidad, $P$ Lámina de agua Iluvia $(\mathrm{mm}), \quad T d$ Duración (h)

Duración (Td) es el tiempo comprendido entre el comienzo y el final de la precipitación que varía según la distribución espacial considerado como evento. Algunos autores consideran que en un sitio específico puede estimarse una sola familia de curvas para un 101 amplio rango de duraciones, por ejemplo entre 5 minutos y 24 horas $^{19}$.

Frecuencia (f) representa el número de veces que se repite una tormenta de características de intensidad y duración definida en un período de tiempo más o menos largo, generalmente en años, se expresa por su periodo de retorno.

La Intensidad Máxima (Imax) es la estimación de la intensidad de precipitación para diferentes duraciones y períodos de retornos necesarios para realizar estimaciones de inundaciones, utilizando los registros de lluvia menores a 24 horas de duración y para regiones hidrológicas muy similares ${ }^{20}$.

En la ecuación 11 se observa la fórmula empírica ${ }^{21}$, la cual es una de las más utilizadas teniendo en cuenta que relaciona la intensidad máxima, con la duración y el periodo de retorno.

$$
I_{\max }=\frac{K T^{a}}{D^{b}}
$$

Ecuacion 11

Dónde $\quad I_{\max }$ - Intensidad Máxima expresada en $\mathrm{mm} / \mathrm{h}, \quad a, b, k$ Constantes obtenidas mediante análisis de regresión múltiple, $T$ Período de retorno (años), $D$ Duración de las precipitaciones (min).

Usualmente para el diseño hidrológico se estudian los caudales máximos presentados durante cada año en las épocas de crecidas. Este análisis se utiliza para caracterizar el conjunto estadístico de los caudales máximos en términos de probabilidades de excedencia. En 
general las estructuras hidráulicas deben ser diseñadas para poder evacuar caudales con probabilidad de excedencia muy pequeña. Para encontrar este caudal de diseño a la serie de caudales máximos se le ajusta una función de distribución de probabilidades teórica, la cual obtiene los caudales máximos con probabilidades de excedencia que aún no han sido registrados en los datos históricos.

La ausencia de datos de caudales es una característica común a la mayoría de pequeños cauces $y$, por ello, su estimación suele hacerse normalmente a partir de la información pluviométrica disponible, mediante alguno de los procesos o métodos conocidos de cálculo.

Dentro de los métodos para el cálculo del caudal máximo se tiene: 1. Método de número de curva, 2. Método del Hidrograma Unitario Triangular

1. Método de Número de Curva fue desarrollado por el Servicio de Conservación de Suelos (SCS) de los Estados Unidos, tiene ventajas sobre el método racional debido a que se aplica a cuencas medianas y pequeñas. El parámetro de mayor importancia de la lluvia generadora es la altura de esta, su intensidad pasa a un segundo plano. Su principal aplicación es la estimación de las cantidades de escurrimiento tanto en el estudio de avenidas máximas como en el caso del cálculo de aportaciones liquidas. El nombre del método deriva de una serie de curvas, cada de las cuales lleva el numero $\mathrm{N}$, que varía de 1 a 100. Un numero de curva $N$ $=100$, indica que toda la lluvia escurre, y un número $N=1$, indica que toda la lluvia se infiltra; por tal razón los números de curvas, representan coeficientes de escorrentía como se aprecia en la ecuación 12.

$Q=\frac{[N(P+50,8)-5080]^{2}}{N[N(P-203,2)+20320]}$

Ecuacion 12

Dónde $Q$ - Escorrentía en $\mathrm{mm}$, $P$ - Lluvía de una determinada duración y período de retorno $(\mathrm{mm}), \quad N$ - Numero de curva

Para el cálculo del caudal máximo se aplica la Ecuación 13.

\section{$Q_{\max }=q \times Q \times A \quad$ Ecuacion 13}

Dónde $\quad Q_{\max }$ - Caudal máximo $\left(\mathrm{m}^{3} / \mathrm{s}\right), \quad q-$ Gasto unitario $\left(\mathrm{m}^{3} / \mathrm{s} / \mathrm{mm} / \mathrm{km}^{2}\right), \quad Q$ - Escorrentía $(\mathrm{mm}), \quad A$ - Área de la cuenca $\left(\mathrm{km}^{2}\right)$

En función de la magnitud o del tiempo de concentración se determina el valor del gato unitario, interpolando linealmente si fuere necesario.

\section{Método del Hidrograma Unitario} Triangular desarrollado por Mockus consiste en un hidrograma unitario sintético de forma triangular, como se muestra en la Imagen 2 que lo usa el SCS (Soil Conservation Service), el cual proporciona los parámetros fundamentales del hidrograma: caudal punta (Qp), tiempo base (tb) y el tiempo en que se produce la punta (tp).

Imagen 2. Hidrograma unitario sintético (forma triangular) 


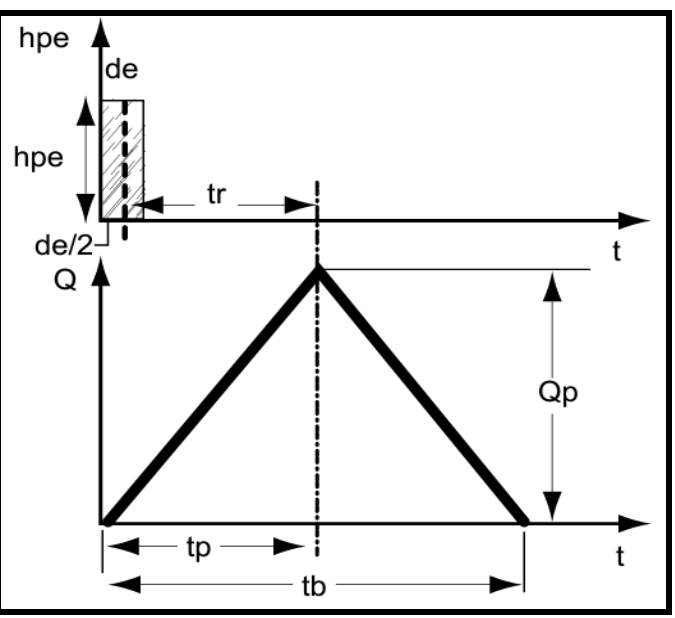

Fuente: Villon, M, 2004

La expresión del caudal punta $\mathrm{Q}_{\mathrm{p}}$, se obtiene igualando el volumen de agua escurrido con el área que se encuentra bajo el hidrograma, mediante las Ecuaciones 14 y 15

$$
\begin{array}{cr}
V e=h p_{e} * A & \text { Ecuacion } 14 \\
V e=\frac{1}{2} * t_{b} * Q_{p} & \text { Ecuacion } 15
\end{array}
$$

Dónde $\quad V e-$ Volumen de agua escurrido, $\quad h p_{a}$ - Altura de precipitación efectiva, $A c-$ Área de la cuenca, $\quad V e-$ Volumen de agua escurrido, $\quad t_{b}$-Tiempo base, $Q_{p}$-Caudal punta

Del análisis de varios hidrogramas, Mockus concluye que el tiempo base y el tiempo pico se relacionan mediante la Ecuación 16 y 17

\section{$t_{b}=2.67 t_{p} \quad$ Ecuacion 16}

$\boldsymbol{t}_{p}=\sqrt{\boldsymbol{t}_{c}}+0.6 \boldsymbol{t}_{c} \quad$ Ecuacion 17 Dónde $t_{b}$-Tiempo base (hr), $t_{p}-$ Tiempo pico (hr), $t_{c}$ - Tiempo de concentración (hr). La duración de exceso (de) con la que se tiene mayor gasto de pico,
103

a falta de datos, se puede calcular aproximadamente para cuencas pequeñas con la Ecuación 18.

de $=t_{c} \quad$ Ecuacion 18

Dónde de-Duración de exceso (hr), $\quad t_{c}$ - Tiempo de concentración (hr)

La Ecuación 19 se obtiene finalmente para realizar el cálculo de caudal punta o caudal máximo en $\mathrm{m}^{3} / \mathrm{s}$.

$Q_{P}=0.208 \frac{h p_{e} * A}{t_{p}}$

Ecuacion 19

\section{METODOLOGIA}

Los datos de precipitaciones máximas mensuales en 24 horas (mms) de la estación La Donjuana 2 con serie histórica 1983 - 2011 fueron suministrados por el IDEAM, luego se calcularon los datos faltantes por el método de proporciones normales para la misma estación. Seguidamente se aplicaron las pruebas de bondad de ajuste Chi - Cuadrado y Kolmogorov - $\quad$ Smirnov, determinando que la distribución de probabilidad a la cual se ajusten los datos es la probabilidad de Gumbel para el cálculo de intensidades máximas. Posteriormente se realizó el cálculo de los parámetros a y $\mu$ para encontrar la probabilidad de ocurrencia según la distribución de Gumbel, asimismo se tuvieron en cuenta los periodos de retorno 2, 5, 10, 20, 25, 50, 75 años, estos fueron seleccionados de acuerdo a las especificaciones técnicas expuestas en el RAS 2000. Luego 
se calcularon las precipitaciones máximas probables.

Con esta precipitación máxima probable y las duraciones que se seleccionaron, se obtiene la intensidad de las Iluvias. Después de este procedimiento se realizó el análisis de regresiones potenciales para cada periodo de retorno con el fin de calcular los parámetros de ajuste a, b, k. Finalmente se obtuvo la ecuación de intensidad máxima con sus respectivos parámetros de ajuste para la microcuenca Aguablanca. Por último, se obtienen las curvas IDF para diferentes duraciones $y$ periodos de retorno.

Se realizaron las salidas de campo a las microcuencas Aguablanca y Chiracoca con el fin de levantar la información espacial de los cauces principales, donde se evidencio que la microcuenca Chiracoca es afluente de la microcuenca Aguablanca. En las salidas de campo se utilizó GPS marca GPSMAP 62s, además se utilizaron los software BaseCamp y ArcGIS 10. De igual manera, se llevaron a cabo charlas con los habitantes de la zona, brindando información sobre eventos climáticos que se han presentado, también se visualizaron puntos de posibles escenarios, zonas con mayor vulnerabilidad a diferentes eventos y se identificó la comunidad que es vulnerable.

Es importante tener en cuenta que no se cuentan con registros de caudal y nivel de agua de ninguna microcuenca, por lo tanto se realizaron aforos en las quebradas La Chiracoca y Aguablanca utilizando el método del correntómetro, con el molinete OTT C 31- Universal. Las mediciones se llevaron a cabo en los meses de septiembre, octubre, noviembre y diciembre, así como también se instalaron escalas hidrométricas en lugares estratégicos para monitorear el nivel de agua, para la estimación del comportamiento del caudal y el nivel del agua en periodos de lluvia y sequía.

Mediante la utilización la imagen satelital identificada con el número $7 / 55$, este se relaciona con la referencia espacial, profundidad del pixel (Pixel Depth) de 8 bit, tamaño de la celda (xy), (Cellsize $(x y)), \quad 28.5, \quad 28.5$ y el procesamiento del software ArcGIS 10 se obtuvieron las características físico - geomorfometricas de la zona de estudio.

Para la determinación de las zonas de inundación se utilizó el software Hec - Ras v. 4.0 y teniendo los caudales máximos para diferentes periodos de retorno. Las fases de trabajo que se llevaron a cabo fueron:

a. Se creó un nuevo proyecto. (File - New Project)

b. Iintroducción de los datos geométricos (Edit - Geometric Data...), se deben dibujar primero las quebradas, las secciones transversales, las cuales se realizaron midiendo profundidades, alturas, bancos, planicies de inundación y longitud aguas abajo de una sección a otra, el coeficiente de Manning, el cual fue seleccionado dependiendo de las condiciones de los bancos y el canal. 
c. Posteriormente se ingresan las condiciones hidráulicas e hidrológicas (caudales y Tiempo de retorno) y condiciones de contorno (Edit - Steady (Unsteady) Flow Data), se crearon 7 de caudales para los respectivos periodos de retorno.

d. Se corre el plan de simulación, de la siguiente forma (Run - Steady (Unsteady) Flow Analysis)

Por último, se creó un plegable en el que se incluyó detalladamente un plan en el que se plasmando temas como: definición de sistema de alerta temprana, objetivo, como funciona, importancia de los planes de difusión de alertas, amenaza, vulnerabilidad y riesgo, capacidad de respuesta de la comunidad, difusión y comunicación de la alerta y significado de las alertas.

\section{RESULTADOS Y DISCUSION}

Las entrevistas, las charlas y los diálogos con la comunidad, permitieron determinar los sitios afectados por fenómenos de deslizamientos e inundaciones; de igual forma, se conoció la percepción de la comunidad sobre la implementación de un SAT. Gran cantidad de habitantes mostraron interés por la implementación del SAT para la protección y seguridad de la comunidad.

De acuerdo a las características físico-geomorfometrico obtenidas se deduce que la microcuenca $Q$. Aguablanca, es casi redonda a oval - redonda moderadamente achatada, con tendencia a la concentración de aguas (formación de crecidas), presentando un desnivel altitudinal mediano, que indica la variedad climática $y$ ecológica que posee. La longitud del cauce es mediana, indicando que el recorrido del agua se tarda un poco en evacuarse. Además está en etapa de equilibrio, es decir, una microcuenca geológicamente madura y es cuenca de pié de montaña

El comportamiento de los datos tiene una distribución normal, presentando una concentración baja de las precipitaciones hacia la media; su curva es asimétrica positiva por lo que los valores se tienden a reunir más en la parte izquierda que en la derecha de la media

De igual manera, se cumple la condición de $x_{c}^{2}<x_{t}^{2}$, por lo tanto los datos de precipitación se ajustan a la distribución normal con un nivel de significación del $5 \%$ de probabilidad y al aplicar el ajuste de Kolmogorov - Smirnov se obtuvo que el delta calculado $(0.1528)$, es menor que el delta teórico $(0.2525)$, entonces el ajuste a los datos de precipitación es bueno a un nivel de significación del $5 \%$, por lo que se infiere que el tipo de distribución Gumbel es ideal para aplicar a las variables analizadas.

En el grafico 1 se observa que las precipitaciones entre 70 y $90 \mathrm{~mm}$ son las que presentan mayor probabilidad de suceder y la menor probabilidad se presenta en entre los intervalos de 90 - 110 y 140 160 respectivamente.

.Grafica 1. Histograma de Frecuencias Relativas de la Microcuenca Aguablanca 


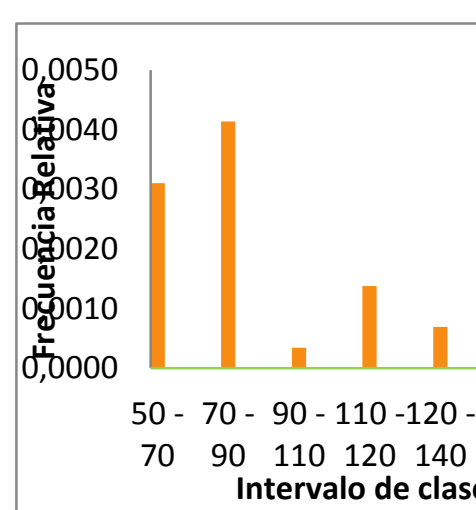

Fuente: Autores.

En la Tabla 2 se obseryan las probabilidades de exceciencia ${ }^{90}$ empíricas según Weibull, 1 con las cuales se obtuvieron los tiempos de retorno para cada uno2 de los eventos máximos. D๑ igual manera, se determinó $₫$ riesgo hidrológico, es decir, 1 si ${ }^{90} \mathrm{la}$ capacidad del

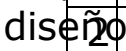
de determinada obra hidráludica ${ }^{100 a}$ superada por un aumento de caudal.

Tabla 2. Cálculo de Probabilidara

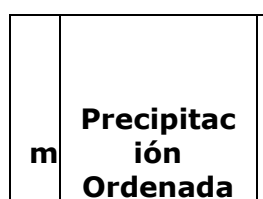

Empírica y Tiempo de Retorno

Probabil

idad

Empíric

a o de

Exceden
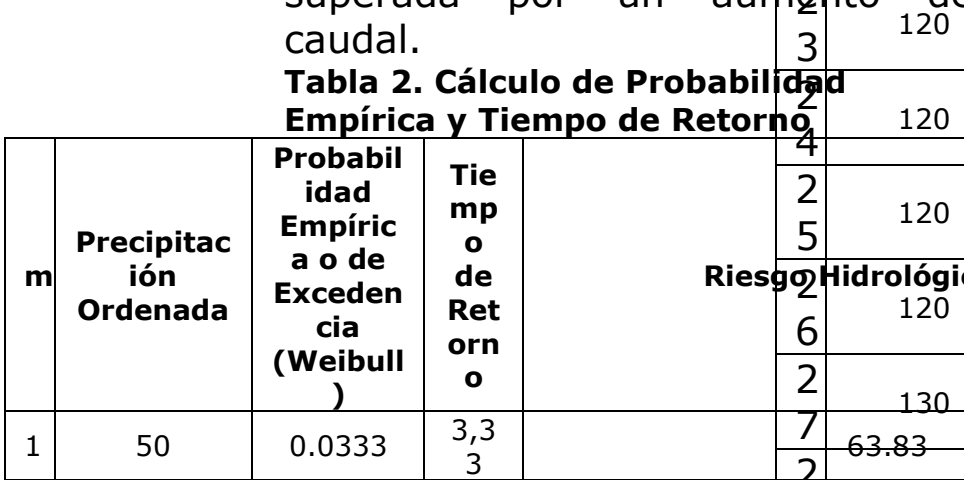

\begin{tabular}{l|l}
\hline 2 & 50
\end{tabular}

350

452

\begin{tabular}{|l|l|} 
& \\
\hline 5 & 60
\end{tabular}

\begin{tabular}{|l|l|}
\hline 6 & 60 \\
\hline
\end{tabular}

\begin{tabular}{l|l}
\hline 7 & 60
\end{tabular}

\begin{tabular}{l|l}
\hline 8 & 66.7
\end{tabular}

\begin{tabular}{l|l}
8 & \\
\hline 9 & 70
\end{tabular}

\begin{tabular}{|l|l|} 
& \\
\hline 1 & 76.6
\end{tabular}
cia (Weibull )

\begin{tabular}{|c|} 
Tie \\
mp \\
o \\
de \\
Ret \\
orn \\
o
\end{tabular}

0.0333

0.0667

0.1000

0.1333

0.1667

\begin{tabular}{|l|r}
\hline 0.2000 & 5 \\
\hline 0.2333 & 4.2
\end{tabular}

0.2333

\begin{tabular}{|c|c}
\hline 0.2667 & $\begin{array}{r}3.7 \\
5\end{array}$ \\
\hline
\end{tabular}

\begin{tabular}{|c|}
\hline 0.3000 \\
\hline 0.3333 \\
\hline
\end{tabular}

Retorng

87.8

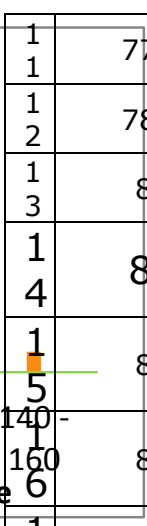

\begin{tabular}{c|c|c|}
0.3667 & 2.7 & 71.23 \\
\hline
\end{tabular}

\begin{tabular}{l|l|l}
0.4000 & 2.5 & 72.11
\end{tabular}

\begin{tabular}{l|c|c}
0.4333 & 2.3 & 73.04
\end{tabular}

\begin{tabular}{l|c|c}
0.4667 & $\begin{array}{c}2.1 \\
4\end{array}$ & 74.00
\end{tabular}

\begin{tabular}{|l|c|c|}
0.5000 & 2 & 75.00 \\
\hline
\end{tabular}

\begin{tabular}{c|c|c}
0.5333 & 1.8 & 76.05 \\
& 8 & 7.05
\end{tabular}

\begin{tabular}{|l|c|c}
0.5667 & $\begin{array}{c}1.7 \\
6\end{array}$ & 77.14 \\
\hline
\end{tabular}

\begin{tabular}{|c|c|c}
0.6000 & $\begin{array}{c}1.6 \\
7\end{array}$ & 78.28 \\
\hline
\end{tabular}

\begin{tabular}{|l|c|c|}
0.6333 & $\begin{array}{c}1.5 \\
8\end{array}$ & 79.49 \\
\hline
\end{tabular}

\begin{tabular}{|l|l|l|}
0.6667 & 1.5 & 80.75 \\
\hline
\end{tabular}

\begin{tabular}{|c|c|c|}
0.7000 & $\begin{array}{c}1.4 \\
3\end{array}$ & 82.09 \\
\hline
\end{tabular}

\begin{tabular}{|c|c|}
0.7333 & $\begin{array}{c}1.3 \\
6\end{array}$ \\
\hline & 1.3
\end{tabular}

\begin{tabular}{|c|c|}
0.7667 & $\begin{array}{c}1.3 \\
0\end{array}$ \\
\hline 0.8000 & $\begin{array}{c}1.2 \\
5\end{array}$ \\
\hline
\end{tabular}

\begin{tabular}{c|c|c|c}
\hline 4 & 120 & 0.8000 & 1.2 \\
\hline 2 & 120 & 0.8333 & 1.
\end{tabular}

gqHidrológico (\%)

\begin{tabular}{|c|c|c|c|c|}
\hline & 12 & 0.833 & 1 & 88.3 \\
\hline & {$\left[\begin{array}{r}\text { Hidroló } \\
12\end{array}\right.$} & $\left(\begin{array}{l}\%) \\
0.866\end{array}\right.$ & 1. & 90.2 \\
\hline & 13 & 0.900 & 1. & 92.2 \\
\hline & \begin{tabular}{l|l}
63.83 \\
& 64.47
\end{tabular} & 0.933 & 1 & 94.5 \\
\hline & 65.13 & 0.966 & 1 & 97.0 \\
\hline
\end{tabular}

$66.51 \quad$ Fuente: Autores.

67.23 Para la equación de la distribución 67.98 de Gumbel se determinaron los parámetros $a=18.35$ y $\mu=76.17$ 69.54 probabilidad de ocurrencia en $70.37 \quad$ distintos periodos de retorno de las
106 
láminas de lluvia, a las cuales se les aplico una corrección de 1.13 dado que el área de la cuenca hidrográfica es de $4308 \mathrm{Ha}$. Luego se obtuvieron los parámetros de ajuste $a, b$ y $c$ y por último se obtuvo la Ecuación 21 de Bernard ajustada para calcular las intensidades máximas para los diferentes periodos de retorno y las duraciones seleccionadas mencionadas.

$I_{\text {máx }}=\frac{329.4 * T^{0,17}}{t^{0,62}}$

Ecuacion 21

Dónde $\quad I_{\text {máx }}$ - Intensidad máxima $(\mathrm{mm} / \mathrm{h}), \quad \mathrm{T}$ - Período de retorno (años), $\mathrm{t}$ - Tiempo de duración de la precipitación (min).

En la Tabla 3 se relacionan la comparación de los caudales máximos obtenidos por cada uno de los métodos aplicados, apreciándose que la variación de un método al otro es aproximadamente de 1 a $5 \mathrm{~m}^{3} / \mathrm{s}$.

Tabla 3. Relación Caudales Máximos Método de Numero de Curva y H.U.T

\begin{tabular}{|c|c|}
\hline $\begin{array}{c}\text { PERIODO DE } \\
\text { REORNO }\end{array}$ & CAUDAL MAXIMO $\begin{array}{c}\text { METODO NUMERO DE CURV } \\
\left(\mathbf{m}^{\mathbf{3}} \mathbf{s} \mathbf{s}\right)\end{array}$ \\
\hline 2 & 6.6786 \\
\hline 5 & 0.53189 \\
\hline 10 & 0.14630 \\
\hline 20 & 2.02771 \\
\hline 25 & 3.06056 \\
\hline 50 & 7.49538 \\
\hline
\end{tabular}

Fuente: Autores.

Las secciones transversales de la Q. Aguablanca que presentaron aumentos de caudales bajo los escenarios de periodos de retorno de 50, 75; 2, 50, 75 años $y$ caudales de $10.907 ; 6.678,7.495$ fueron las números: 1080, 1072 y 1098 respectivamente. La zona de inundación generada para los periodos de retorno de 50 y 75 años es de 2 y 8 m respectivamente, presentándose a la margen izquierda del cauce. De igual manera, las secciones transversales 1037 y 1018 de la Q. Chiracoca presentaron aumentos de caudales bajo los escenarios de periodos de retorno de 50, 75 años y caudales de 7.495, 10.907 respectivamente.

En el Mapa 2 se muestra la ubicación de la zona de estudio resaltando los limites, la red hidrica (Q. Aguablanca, Chiracoca), curvas de nivel, vías de acceso y el casco urbano.

Mapa 2. Ubicación de la Zona de estudio

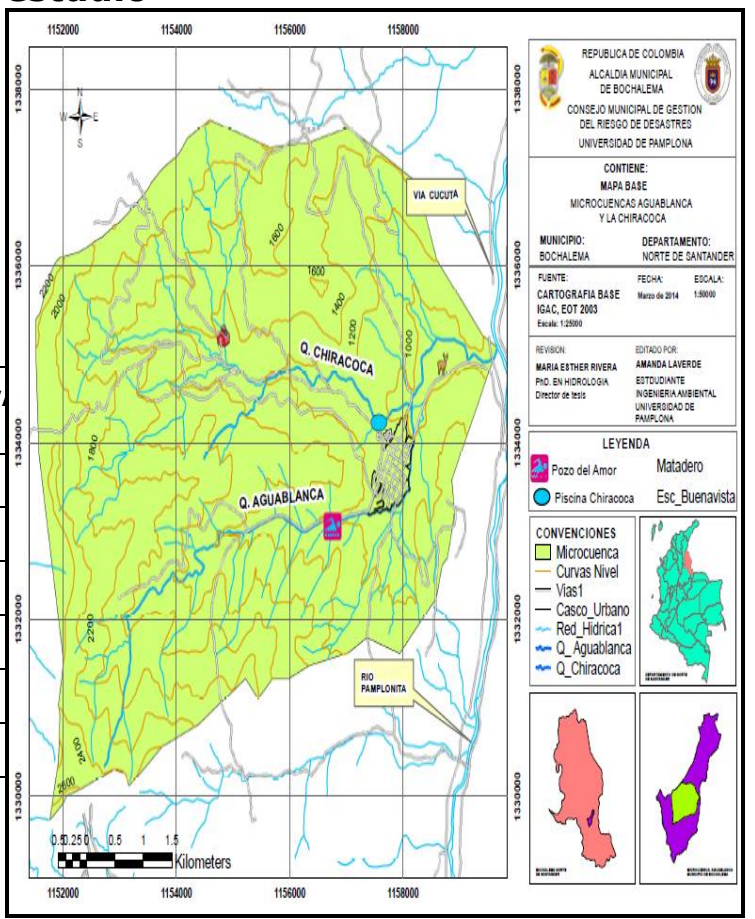

Fuente: Autores.

En el mapa 4 se presenta la información de los sitios más vulnerables en cuanto a eventos extremos: zonas de inundación, 
escarpe con caída de rocas, erosión en cárcavas, terracetas, socavación lateral, reptación, riesgo bajo, moderado y alto con susceptibilidad a inundación. Además, se aprecian tres puntos de inundación en la Q. Aguablanca los cuales corresponden a las secciones 1098, 1080 y 1072 . En la Q. Chiracoca se presentan dos puntos de inundación correspondientes a las secciones 1037 y 1018 . Es necesario resaltar que las secciones nombradas en la anotación anterior se encuentran muy cerca al casco urbano, las cuales se consideran de alto riesgo por la influencia que presentan para la población.

\section{Mapa 4. Riesgos en la zona de estudio}

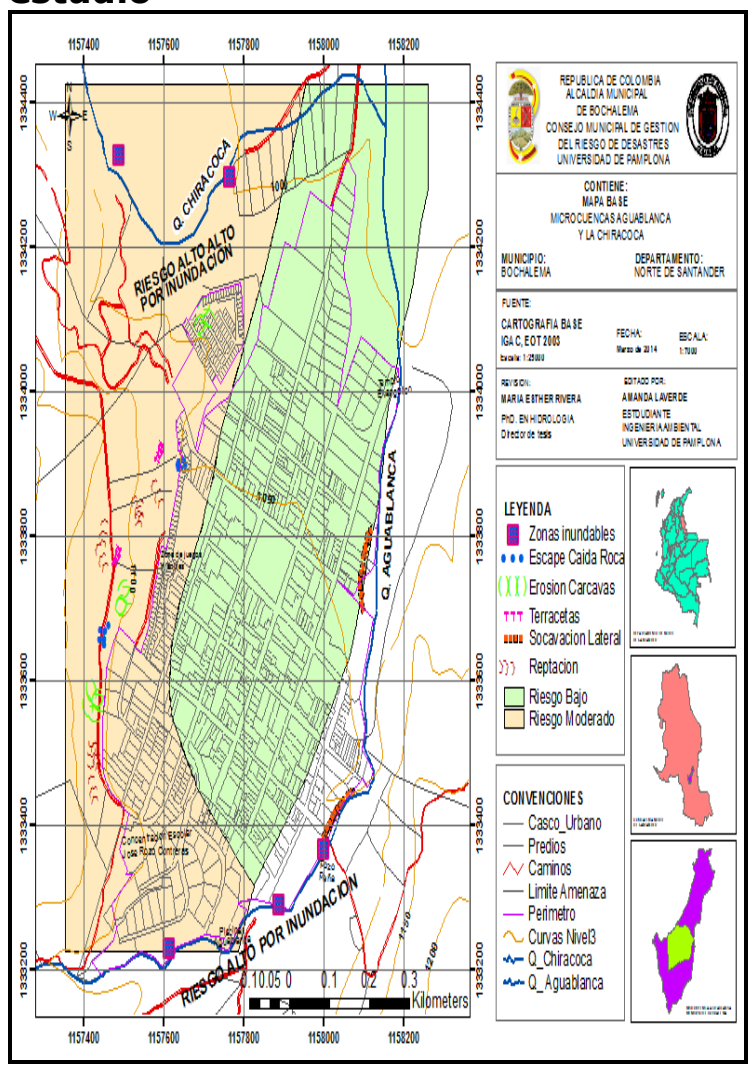

Fuente: Autores
La realización del estudio del montaje institucional y operativo del Sistema de Alerta Temprana (SAT) se llevó a cabo mediante el diagnóstico, del cual se obtuvieron tres zonas que cuentan con las condiciones planteadas según las metodologías utilizadas en los sistemas de alerta internacionales y nacionales.

\section{CONCLUSIONES}

De acuerdo al diagnóstico realizado se obtuvo el lugar óptimo para la ubicación de la estación, se encuentra en la Vereda Zarcuta (cordillera Country Club), pero este lugar se encuentra muy alejado de la zona de estudio por tanto se debe tener en cuenta para la ubicación de la estación la cabecera municipal

Los lugares susceptibles a inundarse y a los que se les debe prestar atención se encuentran en la quebrada Aguablanca y Chiracoca, en las secciones 1072, $1080,1098,1037$ y 1018 msnm respectivamente, con periodos de retorno de 2 a 75 años es importante resaltar que estos puntos son los más cercanos al casco urbano.

Se obtuvieron mapas de la ubicación de la zona de estudio, pendientes y riesgos como herramientas necesarias para llevar a cabo actividades de localización y dar seguimiento a cada amenaza que se presenta en la zona. Mediante la capacitación a la comunidad se logró crear interés en el tema de SAT.

\section{AGRADECIMIENTOS}


Se agradece a la comunidad de la quebrada Aguablanca y Chiracoca de Bochalema por su colaboración en el desarrollo de las mediciones. Al IDEAM por suministrar los datos meteorológicos.

\section{Referencias bibliográficas}

1. Esquema de Ordenamiento Territorial EOT - Bochalema, Norte de Santander. (2003).

2. Organización Meteorológica Mundial. World meteorological organization OMM, "Directrices Sobre Sistemas de Alerta Temprana y Aplicación de Predicción Inmediata y Operaciones De Aviso" Ginebra, Suiza, [internet]. [Consultado en Noviembre de 2011]. Disponible en: http://www.wmo.int/pages/prog/amp/ pwsp/documents/PWS21-

TD1559_111543_es.pdf

3. Organización de los Estados Americanos OEA, "Manual para el Diseño e Implementación de un Sistema de Alerta Temprana de Inundaciones en Cuencas Menores" [internet]. Unidad de Desarrollo Sostenible y Medio Ambiente. Washington, D.C [Consultado en Diciembre de 2011]. Disponible en: http://www.oas.org/dsd/publications/u nit/oea915/MANUAL/.pdf.

4. Administración Nacional Oceánica y Atmosférica NOAA, "Guía De Referencia Para Sistemas de Alerta Temprana de Crecidas Repentinas", National Oceanic and Atmospheric Administration [internet]. [Consultado en Diciembre de 2011]. Disponible en: http://www.meted.ucar.edu/communit es/hazwarnsys/ffewsig es/FFG comple ta es.pdf 109
5. Aristizábal, E., Gamboa, M. F., y Leoz, F. J. 2010. Sistema de alerta temprana por movimientos en masa inducidos por lluvia para el Valle de Aburrá, Colombia, Revista EIA, 7 (13), 155-169 [internet]. [Consultado 2012 Enero]. Disponible en: http://hdl.handle.net/11190/189

6. Fernández L, Sabas R, C Andrés. 2011. Sistema de Alerta Temprana Centrado en la Población para la Cuenca Media del Río Otún [internet], Universidad Tecnológica de Pereira, (Colombia). [Consultado Eenero de 2012]. Disponible en: http://revistas.utp.edu.co/index.php/r evistaciencia/article/view/1583/3967

7. Mejía Fernández $F$, Londoño Linares J P, Pachón Gómez, J A, "Red de Estaciones Meteorológicas para Prevención de Desastres en Manizales - Caldas" [internet], Universidad Nacional de Colombia de Manizales (Colombia), [Consultado en Diciembre de 2011]. Disponible en: http://idea.manizales.unal.edu.co/gest ion_riesgos/descargas/gestion/redesta ciones.pdf

8. Organización de los Estados Americanos OEA, "Manual para el Diseño e Implementación de un Sistema de Alerta Temprana de Inundaciones en Cuencas Menores" [internet]. Unidad de Desarrollo Sostenible y Medio Ambiente. Washington, D.C [Consultado en Diciembre de 2011]. Disponible en: http://www.oas.org/dsd/publications/u nit/oea915/MANUAL/.pdf.

9. Segerer, C. D., Villodas, R. 2007. Hidrología I. Unidad 9: Estadística aplicada a la Hidrología. Universidad Nacional de Cuyo. Ingeniería Civil. Argentina.

10. Amaro I, Demey J, Macchiavelli R. Aplicación del análisis $\mathrm{r} / \mathrm{s}$ de hurst para 
estudiar las propiedades fractales de la precipitación en Venezuela. Interciencia, 29:617-620. 2004.

11. Takens, F. Detecting strange attractors in turbulence. In: Rand DA, Young LS (Eds.), Dynamical Systems and Turbulence, Lecture Notes in Mathematics, 898:366-381, SpringerVerlag Takens, F (1985). On the numerical determination of the dimension of the attactor. In Dynamical Systems and Bifurcations, Lecture notes in mathematics, 1125:99-106, Springer-Verlag. Citado por Infante S, Ortega J. y Cedeño F. Estimación de datos faltantes en estaciones meteorológicas de Venezuela vía un modelo de redes neuronales. Revista de Climatología. Vol. 8 (2008): 51-70 ISSN 1578-8768. [Consultado en Octubre de 2011]. Disponible en: http://webs.ono.com/reclim3/reclim08 e.pdf

12. Villón, M. V. 2006. Hidrología Estadística. Costa Rica: Tecnológica de Costa Rica.

13. Chow, Ven Te. 1994. Hidrología Aplicada. Santafé de Bogotá, Colombia: Mc-Graw Gill, Interamericana S.A.

14. Hershfield, D.M., Weiss, L.L., y Wilson, W.T., 1955: Synthesis of rainfall intensity regimes. Proceedings of the American Society of Civil Engineers, Volumen 81 , Separata No. 744. [Consultado en Octubre de 2011]. Disponible en: http://documentacion.ideam.gov.co/op enbiblio/bvirtual/012406/Cap28.pdf

15. Koutsoyiannis, D. Kozonis, D. y Manetas, A. 1998. A mathematical framework for studying rainfall intensity-duration-frequency

relationships. Journal of Hydrology 206: 118-135
16. Smith, J.A., 1993. Handbook of Hydrology, capítulo III, Precipitation, McGraw-Hill, Inc. primera edición, USA, p. 1-47.

17. Willems, P., 2000. Compound intensity/duration/frequencyrelationships of extreme precipitation for two season and two storm types. Journal of Hydrology, vol. 233, p.189205 Citado por Domitilo Pereyra-Díaz, José Antonio A. Pérez-Sesma y Leonorilda Gómez-Romero. Ecuaciones que Estiman Las Curvas IntensidadDuración-Período de Retorno de la Lluvia. GEOS, 2004, Vol. 24, No. 1, p. 46-56. [Consultado en Octubre de 2011]. Disponible en: http://www.ugm.org.mx/publicaciones /geos/pdf/geos04-1/Pereyra-

Diazetal.pdf

18. Chow, Ven Te, Maidment, David R. y Mays, Larry W 1994. Hidrología Aplicada. Capitulo Análisis de Frecuencias. Santafé de Bogotá, Colombia: Mc-Graw Gill, Interamericana S.A.

19. Bonacci, O. 1984. Rainfall as the basis urban-runoff- Experience and practice in Yugoslavia. Water Science and Technology vol $16 \mathrm{~N}^{\circ}$ 8-9 pp 102108.

20.Kothyari, U. and Garde, R. (1993). "Closure to "Rainfall Intensity-Duration-Frequency Formula for India" by Umesh C. Kothyari and Ramachandra J. Garde (February, 1992, Vol. 118, No. 2)." J. Hydraul. Eng., $10.1061 /($ ASCE) 07339429(1993)119:8(966), 966-967. [Consultado en Noviembre de 2011]. Disponible en: http://ascelibrary.org/doi/abs/10.1061 L(ASCE)0733-9429(1993)119:8(966) 
21. Bernard, M.M., 1932. Formulas for rainfall intensities of long durations.

Trans. ASCE, vol. 96, p. 592-624

\section{Laverde P Luz Amanda.}

Ingeniero Ambiental. Facultad de Ingenierías y Arquitectura. Programa Ingeniería Ambiental. Grupo de Investigaciones Ambientales Agua, Aire y Suelo (GIAAS). Universidad de Pamplona. Pamplona. Colombia

\section{Rivera María Esther.}

Ph.D Hidrología .Profesor Asociado.Facultad de Ingenierías y Arquitectura. Programa Ingeniería Ambiental. Grupo de Investigaciones Ambientales Agua, Aire y Suelo (GIAAS). Universidad de Pamplona. Pamplona. Colombia. 